\title{
The Effects of Walking and Control Method on Pressure-Based Interaction
}

\author{
Graham Wilson \\ Stephen Brewster \\ Martin Halvey \\ Glasgow Interactive Systems \\ Group \\ School of Computing Science \\ University of Glasgow \\ Glasgow, G12 8QQ UK \\ gawilson@dcs.gla.ac.uk \\ Stephen.Brewster@glasgow.ac.uk \\ Martin.Halvey@glasgow.ac.uk
}

\begin{abstract}
Pressure-based interactions have largely been limited to static scenarios; very few have focused on its use on mobile devices and even fewer have investigated the use of pressure while the user is in motion (i.e. walking). This paper presents the initial results of research looking into the effects of walking on the application of pressure during linear targeting. Positional and ratebased (velocity) control methods are compared in order to determine which allows for more stable and accurate selections. Results suggest that rate-based control is superior for both mobile (walking) and static (sitting) linear targeting and that mobility significantly increases errors, selection time and subjective workload. These results will influence the design of a second part of the study, which will evaluate user ability to control the same application using only audio feedback.
\end{abstract}

\section{Keywords}

Pressure input, mobile interaction, non-visual feedback.

\section{ACM Classification Keywords}

H5.2. User interfaces: Haptic IO.

Copyright is held by the author/owner(s)

CHI 2011, May 7-12, 2011, Vancouver, BC, Canada.

ACM 978-1-4503-0268-5/11/05.

\section{General Terms}

Design, Performance. 


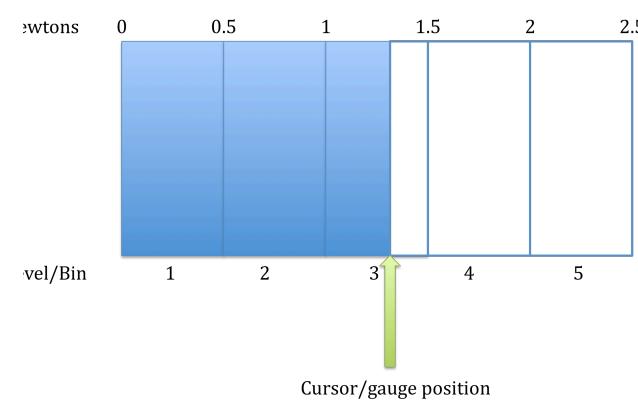

Figure 1: Example linear targeting implementation. Cursor position is dictated by applied force (positional control).

\section{Introduction}

Pressure has been used for many different applications from shape-translation [5] to password security ${ }^{1}$. The most common use by far, however, is linear (1dimensional) targeting. In this type of application the interaction space (referred to as pressure space) is divided into a number of bins or levels of equal width (in Newtons) and the user applies pressure to reach a target level (see Figure 1). Making the target levels thinner gives an indication of both how accurately/precisely users can apply pressure and how many items can be used in a pressure-based interaction (e.g. for menus, zoom level, etc.). However, there are important aspects missing from this body of research including the use of alternative control methods and how mobility can influence the ability to control pressure.

\section{Pressure Input}

Research has suggested that users can accurately acquire between 6 [4] and 10 [7] levels of pressure, with several influencing factors including the pressure sensor being used, how the sensor signal is treated and the feedback being provided. Several studies suffered due to non-linear output from the pressure sensor used. Ad hoc software treatments of the signal somewhat mitigated these issues, but using a properly linearised sensor output, like that developed by Stewart et al. [6], facilitates high levels of accuracy. Using this sensor, Wilson et al. [7] found that users were able to use up to 10 levels of pressure at $97-100 \%$ accuracy while sitting, and up to $80 \%$ accuracy using only audio feedback.

\footnotetext{
${ }^{1}$ http://jdadesign.net/?page_id=37
}

\section{Control Methods and Mobility}

Nearly all research that has used linear targeting to judge our ability to control pressure has used a control method called positional control, where the gauge fills (or a cursor moves through the levels) as the user applies more pressure. Releasing pressure moves the gauge/cursor back to the beginning (as in Figure 1). An alternative control method is rate-based or velocity control, where the speed of cursor movement is controlled by the amount of force applied, so that low forces move the cursor slowly and high forces move it quickly. Shi et al. [5] found that rate-based input allowed for superior control of pressure used in a shape translation task when users were seated at a desk.

Crossan et al. [2] found that head tilt using rate-based control produced better accuracy than positional, at the cost of slower selection time, when users were walking. Conversely, they found that positional control allowed for superior accuracy and selection time when users were sitting.

The majority of pressure input research has been carried out in static settings with users seated at a desk using pressure-sensitive styli or Force-Sensing Resistors (FSR) attached to other input devices (e.g. a mouse). A few studies have looked at control of pressure on mobile devices but have tested only positional control of the interaction. Only one of these studies has actually involved the user moving and looked at how mobility influences input effectiveness [1]. This study found that walking negatively affected accuracy when selecting 2 levels of pressure. Other work looking at how walking influences mobile interaction has also found negative effects on control accuracy when tapping on-screen with a finger [3] and during linear targeting via head tilt [2]. 


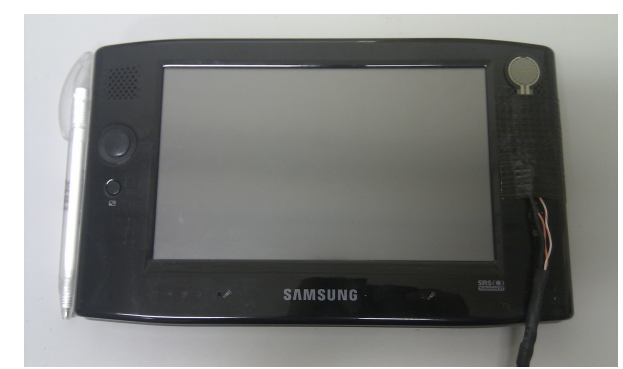

Figure 2: Samsung UMPC model Q1 with FSR attached (top right).

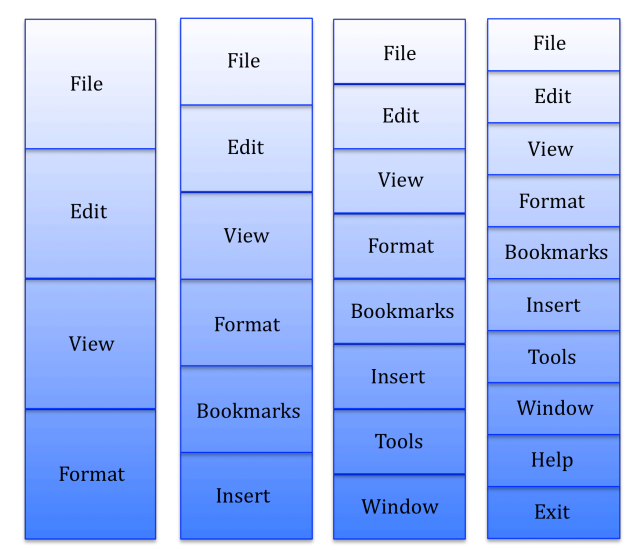

Figure 3: Menu layouts for the 4, 6, 8 and 10 item menus with relative target sizes.
We therefore wanted to investigate 1) how mobility influences our ability to control pressure; 2) which control method, positional or rate-based, allows for more stable and accurate control of pressure when mobile; and 3) whether this knowledge can be used to design non-visual mobile pressure interaction as it has been shown that users can accurately apply pressure using only audio feedback while sitting [7].

\section{Experimental Design}

The whole experiment was divided up into two subexperiments/sessions. The purpose of the first session was to establish which of the control methods allowed for better control of pressure input while the user is walking, before the second session used this superior method and asked users to interact with the application using only audio feedback. The paper here covers some of the results from the first session.

\section{Evaluation}

\section{Apparatus}

Pressure sensing was achieved via an Interlink Electronics Force-sensing resistor (FSR) model 402 FSR (see Figure 2, top right). The FSR was connected to an SAMH Engineering ${ }^{2}$ SK7-ExtGPIO1 input/output module for A-to-D conversion and output to host machine.

Within the firmware of the IO module was the linearising firmware developed by Stewart et al. [6]. The experimental software ran on a Samsung Q1 UMPC (see Figure 2). The FSR was attached to the bezel of the device on the left or right hand side near the top, depending on the participant's dominant hand.

\section{Experimental Task}

The task used was identical to that used by Wilson et al. [7], itself being a design based on previous linear targeting implementations. A pressure-space of approximately $4 \mathrm{~N}$ was used, with the space divided up into $4,6,8$ or 10 equal-sized bins or pressure levels. The whole space was visualized on-screen as a vertical menu of $100 \times 400$ pixels, running from top-to-bottom of the screen, containing either 4, 6, 8 or 10 menu items (see Figure 3). Each item was given a text label and both the labels and the layout of the menus were similar to those found in many common mobile and desktop applications. Each trial involved selecting a single target menu item: the participant had to move a cursor down the menu using pressure so that it was within the boundaries of the target item before selecting it via a 1 -second Dwell technique. Dwell has been shown to be the most accurate in linear targeting, although it suffers longer overall targeting time due to the imposed dwell duration $[1,4]$.

\section{Control Methods}

POSITIONAL CONTROL

In this method the position of the cursor in the menu is dictated by how hard the participant presses on the FSR (like in Figure 1). As the pressure space was approximately $4 \mathrm{~N}$, applying and maintaining $1 \mathrm{~N}$ would hold the cursor approximately $1 / 4$ of the way down the menu, $2 \mathrm{~N}$ would hold it half way and so on. As the menu and task were designed to have a degree of realworld relevance, it was impossible to move the cursor past the bottom of the menu. Target selection was achieved by maintaining a level of pressure that held the cursor within the target item for 1 second.

\footnotetext{
${ }^{2}$ www.samh-engineering.com, stephenahughes@gmail.com
} 


\begin{tabular}{|c|c|}
\hline $\begin{array}{c}\text { Approx. } \\
\text { Pressure (N) }\end{array}$ & $\begin{array}{c}\text { Speed } \\
\text { (pix (mm)/s) }\end{array}$ \\
\hline 0 & Stationary \\
\hline 0.2 & $33(6.6)$ \\
\hline 0.4 & $66(13.2)$ \\
\hline 0.6 & $99(19.8)$ \\
\hline 0.8 & $132(26.4)$ \\
\hline 1.0 & $165(33)$ \\
\hline 1.2 & $198(39.6)$ \\
\hline 1.4 & $231(46.2)$ \\
\hline 1.6 & $264(52.8)$ \\
\hline 1.8 & $297(59.4)$ \\
\hline 2.0 & $330(66)$ \\
\hline
\end{tabular}

Table 1: Rate-based condition speeds in pixels and $\mathrm{mm}$ per second. based on pressure input.
RATE-BASED CONTROL

In this method the velocity of the cursor's downward motion was dictated by how hard the participant pressed on the FSR, with no pressure bringing it to a halt. Initial pilot testing led to the adoption of ten 'speeds', up to a maximum of 330 pixels, or $66 \mathrm{~mm}$, per second (see Table 1). This design only allowed for downward motion of the cursor. Although a second FSR could have been used to allow for upward motion (in the case of overshooting a target), the positional control method only utilized one sensor, so it was decided to use only one for rate-based control also, to keep the interactions as similar as possible. Therefore, if the participant overshot a target, they could push the cursor past the bottom of the menu and it would 'loop' back to the top of the menu and start again. Target selection was achieved by stopping the cursor (applying no pressure) within the item and leaving it stationary for 1 second.

\section{Mobility}

During the static condition, participants were seated in a padded office chair holding the UMPC in both hands. They were allowed to rest their arms on either their legs/knees or a desk in front of them to provide stability, but could not rest their wrists or the device while interacting. The mobile condition used a similar design to Crossan et al. [2] as it requires divided attention between task and navigation. Participants were asked to walk in a marked $4 \mathrm{~m} \times 3 \mathrm{~m}$ figure-of-eight route indoors while they interacted with the device.

Participants \& Experimental Procedure Fourteen participants (11 male, 3 female) aged between 17 and 30 years old (mean $=22.8$ ) took part in the evaluation, all of whom were studying or working at the University. 13 were right-handed and all were paid $£ 20$ for participation in both parts of the study.

The experiment was divided into four conditions so that participants completed two static and two mobile conditions, one using each of the control methods: StaticPositional, Static-Rate, Mobile-Positional and MobileRate. The order of these 4 conditions was counterbalanced to avoid order effects. Within each condition every menu item from all four of the menu sizes $(4,6$, 8 and 10 items) was to be selected twice. The presentation order of menu sizes was randomized, and all targets within that menu were presented in a random order. Participants completed a NASA TLX workload estimation form after each condition.

\section{VARIABLES}

There were three Independent Variables: Control Method (Positional, Rate-based), Mobility (Sitting, Walking) and Menu size (4, 6, 8 or 10).

Dependent Variables were: Errors (ER; whether the correct menu item was selected), Movement Time (MT time between first non-0 pressure value and selection, be that correct or incorrect), Number of Crossings (NC; the number of times the cursor crossed either of a target item's boundaries) and Workload measured via the NASA TLX. NC has been used as an indication of control, as a lower number suggests more stable and precise application of pressure, however it applies only to positional control. A somewhat similar measure, here called Loops, was used for rate-based conditions and measured the number of overshot attempts. We also used a SHAKE sensor device to detect a users motion while they walked via its inbuilt accelerometer. 


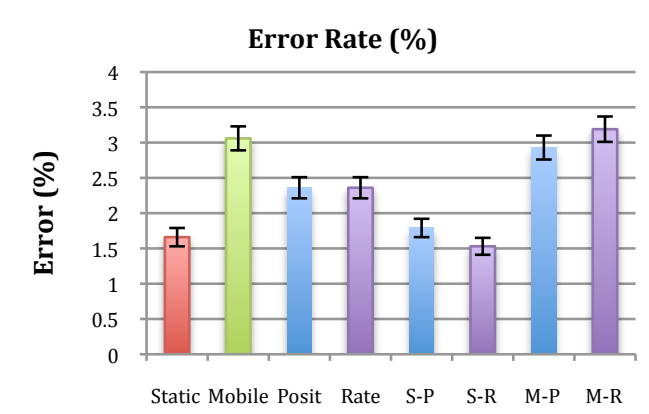

Condition

Figure 4: Mean error rates and SD tor each condition.

\section{Results}

Errors

A Wilcoxon pairwise comparison showed a significant effect of mobility on number of errors ( $T=682.50$, $\mathrm{p}<.01$ ), as walking (mean $=3.1 \%$ ) produced more errors than sitting (mean=1.7\%). There was no effect of control method on errors $(T=1242.50, p>.05)$ as both had ER of $2.4 \%$. Comparing conditions, Wilcoxon $T$ pairwise comparisons showed a significant difference between the Static-Rate and the Mobile-Rate conditions $(T=170, p<.05)$. All other comparisons were not significant $(p>.05)$. Error rates for the four conditions (see figure 4) were: $1.8 \%$ for Static-Positional ( $S D=0.13)$, $1.5 \%$ for Static-Rate (SD=0.12), $2.9 \%$ for MobilePositional (SD=0.17) and $3.2 \%$ for Mobile-Rate $(\mathrm{SD}=0.18)$.

\section{Movement Time}

Both mobility $(T=472356.5, \mathrm{p}<.001)$ and control method ( $T=266186.5, \mathrm{p}<.001)$ significantly affected control of pressure, with rate-based control (mean $=2.29 \mathrm{~s}$ ) allowing for faster selections than positional control (mean=3.37s) and walking (mean=3.11s) causing slower selections than sitting (mean=2.55s). Wilcoxon $T$ comparisons found that all conditions were significantly different from each other $(p<.001)$, with the exception of Static-Rate vs MobileRate $(p>.05)$. Mean movement times for each condition (including 1s Dwell time) were 2.85s (Static-Positional), 2.24s (Static-Rate), 3.88s (Mobile-Positional) and 2.34s (Mobile Rate; see Figure 5).

\section{Number of Crossings/Loops}

$\mathrm{NC}$ only applies to the positional control method and so only Static-Positional and Mobile-Positional were compared. Wilcoxon pairwise comparison showed that mo- bility had a significant effect on the number of crossings $(T=170, p<.05)$ with mobile selections resulting in more crossings, suggesting a lower degree of control. Loops only applied to the Static-Rate and Mobile-Rate conditions and there was a significant effect of mobility found on the number of overshoots (Wilcoxon $T=39.50$, $\mathrm{p}<.05$ ) with mobile selections producing significantly more overshoots per selection (mean $=0.46)$ than static selections $($ mean $=0.12)$.

\section{Subjective Workload}

Repeated-measures ANOVA showed that being mobile significantly increased overall subjective workload compared to sitting $\left(F_{1,13}=16.103, p<.05\right.$; see figure 6$)$. Looking at individual measures, mobility significantly increased subjective reports of Mental Demand $\left(F_{1,13}=\right.$ $27.161, \mathrm{p}<.05)$, Physical Demand $\left(\mathrm{F}_{1,13}=58.018\right.$, $\mathrm{p}<.05)$, Effort required $\left(\mathrm{F}_{1,13}=85.018, \mathrm{p}<.001\right)$, and Frustration $\left(F_{1,13}=39.446, p<.05\right)$. It also significantly worsened perceived performance $\left(F_{1,13}=31.5, p<.05\right)$.

Rate-based control significantly reduced overall workload $\left(F_{1,13}=78.381, p<.01\right)$ as well as subjective reports of Mental Demand $\left(F_{1,13}=204.446, p<.01\right)$, Physical Demand $\left(F_{1,13}=135.161, p<.01\right)$ and Effort required $\left(F_{1,13}=196.875, p<.01\right)$. All other effects were not significant $(p>.05)$.

\section{Discussion}

The analysis carried out so far suggests that Ratebased input allows for superior control of pressure compared to positional input for both static and mobile interaction. Although both control methods enjoyed equal accuracy, rate-based selections were significantly faster when both sitting and walking and were rated as significantly less mentally and physically demanding.
Figure 5: Mean selection time and SD for each condition. 


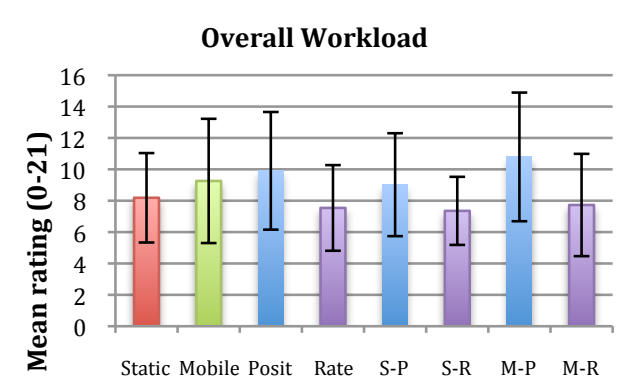

Condition

Figure 6: Mean and SD for NASA TLX Workload ratings.
Therefore this control method appears to be better than the standard method used in linear targeting research, with mobile rate-based selections even being faster than static positional ones. Mean ER and MT for StaticPositional selections are similar to those found for the same condition in Wilson et al [7], ER being equal, but MT being approximately $0.4 \mathrm{~s}$ slower on average in this study.

Walking had a big impact on user performance, producing more errors and taking, on average, one second longer per selection. It also greatly increased workload and frustration levels. Although NC and Loops are not correlate measures, the higher values produced when walking indicate a lower degree of control during mobile selections. Mobility appears to have a smaller impact on rate-based selection time than on positional selection time, however. Walking increased average positional selection time by $1.02 \mathrm{~s}$ but only increased it by $0.1 \mathrm{~s}$ under rate-based control. Therefore use of rate-based input may mitigate the negative effects of mobility to a degree.

\section{Conclusions and Future Work}

From these initial results it appears that mobility has a significant negative influence on control of pressure, particularly on positional control. Rate-based input is therefore best suited to mobile control of pressure, at least when used for linear targeting. Of particular interest is the finding that rate-based control allows for faster and equally accurate linear targeting selections than the common positional method used in most research. Therefore the rate-based method will be used during the second session, which will investigate whether users are able to interact with this application using only audio feedback. Non-visual interaction is an important facet of mobile device use, to ease interaction when navigating in the environment and to reduce reliance on relatively small screen space. Missing from the analysis here is data concerning walking speed and body movement and the impact of these on interaction (and the effect of the interaction on walking speed). Gait phase analysis [3] will allow us to analyse at what points users change their walking speed, if they do at all, and whether stepping introduces involuntary and unwanted influences on the input.

\section{Acknowledgements}

This research was jointly funded by the industrial members of MobileVCE (www.mobilevce.com) and EPSRC grant EP/G063427/1.

\section{References}

[1] Brewster, S. and M. Hughes. Pressure-Based Text Entry for Mobile Devices. In MobileHCI '09, Bonn, Germany, 2009.

[2] Crossan, A., M. McGill, S. Brewster, and R. MurraySmith. Head Tilting for Interaction in Mobile Contexts. in MobileHCI '09. 2009. Bonn, Germany.

[3] Crossan, A., R. Murray-Smith, and S. Brewster. Gait phase effects in mobile interaction. in $\mathrm{CHI}^{\prime} 05$ Extended Abstracts. 2005. Portland, Oregon.

[4] Ramos, G., M. Boulos, and R. Balakrishnan. Pressure widgets. In Proceedings of CHI 2004, Vienna, Austria, 2004.

[5] Shi, K., S. Subramanian, and P. Irani. PressureMove: Pressure Input with Mouse Movement. IFIP INTERACT 09, 2009.

[6] Stewart, C., M. Rohs, S. Kratz, and G. Essl. Characteristics of Pressure-Based Input for Mobile Devices. In Proceedings of CHI 2010, Atlanta, Georgia, 2010.

[7] Wilson, G., C. Stewart, and S. Brewster. PressureBased Menu Selection for Mobile Devices. In MobileHCI '10, Lisbon, Portugal, 2010. 